\title{
L3MBTL2 regulates chromatin remodeling during spermatogenesis
}

\author{
Chenling Meng ${ }^{1} \cdot$ Jinyue Liao ${ }^{1} \cdot$ Danfeng Zhao $^{2} \cdot$ Huihui Huang ${ }^{1} \cdot$ Jinzhong Qin ${ }^{3} \cdot$ Tin-Lap Lee $^{1} \cdot$ Degui Chen $^{2} \cdot$ \\ Wai-Yee Chan ${ }^{1,4,5} \cdot$ Yin Xia $\mathbb{B}^{1,4,5}$
}

Received: 12 August 2018 / Revised: 18 December 2018 / Accepted: 7 January 2019 / Published online: 13 February 2019

(c) ADMC Associazione Differenziamento e Morte Cellulare 2019

\begin{abstract}
Lethal (3) malignant brain tumor like 2 (L3MBTL2) is a member of the MBT-domain proteins, which are involved in transcriptional repression and implicated in chromatin compaction. Our previous study has shown that L3MBTL2 is highly expressed in the testis, but its role in spermatogenesis remains unclear. In the present study, we found that L3MBTL2 was most highly expressed in pachytene spermatocytes within the testis. Germ cell-specific ablation of L3mbt12 in the testis led to increased abnormal spermatozoa, progressive decrease of sperm counts and premature testicular failure in mice. RNAsequencing analysis on L3mbtl2 deficient testes confirmed that L3MBTL2 was a transcriptional repressor but failed to reveal any significant changes in spermatogenesis-associated genes. Interestingly, L3mbtl2 deficiency resulted in increased $\gamma \mathrm{H} 2 \mathrm{AX}$ deposition in the leptotene spermatocytes, subsequent inappropriate retention of $\gamma \mathrm{H} 2 \mathrm{AX}$ on autosomes, and defective crossing-over and synapsis during the pachytene stage of meiosis I, and more germ cell apoptosis and degeneration in aging mice. L3MBTL2 interacted with the histone ubiquitin ligase RNF8. Inhibition of L3MBTL2 reduced nuclear RNF8 and ubH2A levels in GC2 cells. L3mbtl2 deficiency led to decreases in the levels of the RNF8 and ubH2A pathway and in histone acetylation in elongating spermatids, and in protamine 1 deposition and chromatin condensation in sperm. These results suggest that L3MBTL2 plays important roles in chromatin remodeling during meiosis and spermiogenesis.
\end{abstract}

\section{Introduction}

Spermatogenesis is composed of three phases: mitosis of the spermatogonia, meiosis of spermatocytes, and differentiation of round spermatids into mature spermatozoa. The third phase is also known as spermiogenesis [1]. During meiosis, homologous chromosomes undergo recombination. Double strand DNA breaks (DSBs) are generated during the prophase of meiosis I to allow for homologous recombination and crossing over to take place. DSBs trigger the DNA damage response, resulting in recruitment of $\gamma \mathrm{H} 2 \mathrm{AX}$ and many other components of DNA damage response machinery. DNA repair then occurs, which is accompanied with synapsis of homologous autosomal chromosomes, and

Edited by V D'Angiolella

Supplementary information The online version of this article (https:// doi.org/10.1038/s41418-019-0283-z) contains supplementary material, which is available to authorized users.

Yin Xia

Xia.Yin@cuhk.edu.hk

Extended author information available on the last page of the article reciprocal recombination between homologs [2-6]. However, how DSBs and the subsequent DNA repair are regulated during the prophase of meiosis I are not fully understood.

During spermiogenesis, round spermatids undergo a number of important changes including elongation and condensation of the nucleus. The morphological changes in the nuclear content of spermatids require histone acetylation. Acetylated histones then are replaced by the transition proteins TNP1 and TNP2 and subsequently by protamines 1 and 2, which package the sperm genome into a distinct toroid chromatin structure [7-12]. Despite the importance of histone-to-protamine transition in male fertility, it is largely unknown how this transition is triggered. Recent studies suggest that $\mathrm{H} 2 \mathrm{~A}$ and $\mathrm{H} 2 \mathrm{~B}$ ubiquitination mediated by the E3 ubiquitin ligase RNF8 in elongating spermatids is a key initial step in $\mathrm{H} 4$ acetylation and histone removal [13, 14], but the regulatory mechanisms underlying activation of the RNF8-ubH2A pathway have just begun to be elucidated.

Lethal (3) malignant brain tumor like 2 (L3MBTL2) is a member of the MBT protein family composed of nine members in humans and mice $[15,16]$. These proteins are characterized by the presence of tandem arranged two, three or four 100-amino acid MBT domains. The MBT domains 

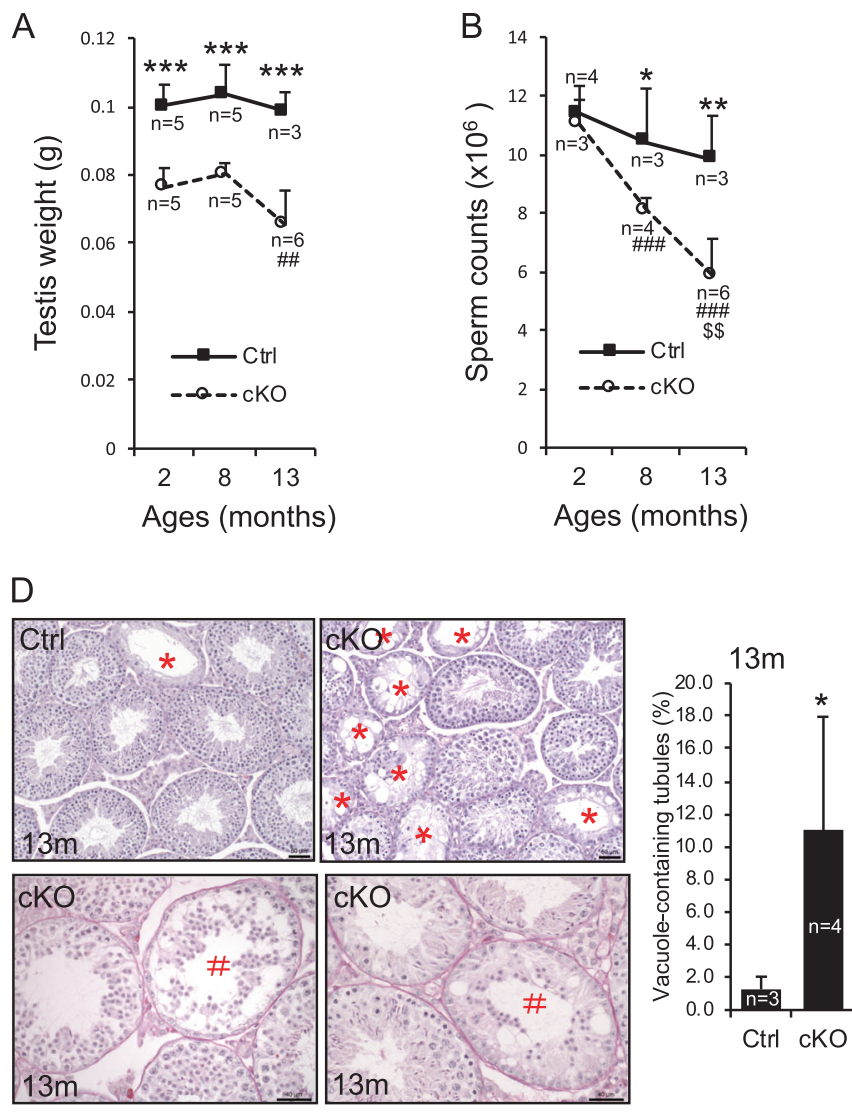

Fig. 1 Deletion of L3mbt12 leads to reduced sperm counts and spermatogenic defects. a Testis weights in control (L3mbtl $\left.2^{\mathrm{f} / \mathrm{f}}\right)$ and L3mbtl2 cKO (L3mbtl2 ${ }^{\mathrm{f} / \mathrm{f}}-\mathrm{Stra} 8$-icre) mice at 2,8 , and 13 months of age. The average of the paired testes of each mouse was used. $\mathbf{b}$ Number of sperms per caudal epididymis from control and L3mbtl2 cKO mice at 2, 8, and 13 months of age. The average of the two caudal epididymides of each mouse was used as the number of sperms per caudal epididymis. c PAS-stained cross sections of seminiferous tubules from 2- and 8-month-old control and L3mbtl $2 \mathrm{cKO}$ mice. Red arrows indicate multinucleated cells. Red asterisks represent tubules with loss of germ cells. d PAS-stained cross sections of seminiferous tubules from 13-month-old control and L3mbtl $2 \mathrm{cKO}$ mice. The ratios

were first identified in the Drosophila tumor-suppressor protein L(3)MBT, and mutations of the $L(3) m b t$ gene lead to development of lethal malignant brain tumor in the larva [17]. In humans and mice, two proteins, SCMH1 and SCML2, contain two MBT domains. Three proteins, L3MBTL1, L3MBTL3, and L3MBTL4, have three MBT domains. Finally, four proteins, L3MBTL2, MBTD1, SFMBT1, and SFMBT2, contain four MBT domains [15, 18].

MBT domain proteins participate in the modification of chromatin architecture by binding histones and compacting chromatin [15, 19, 20]. L3MBTL1 compacts oligonucleosomal arrays only in the presence of methyl marks, such as mono- and dimethylated H4K20 and H1bK26 $[20,21]$. In contrast to L3MBTL1, L3MBTL2 binds and compacts nucleosomes independently of histone modifications [22].

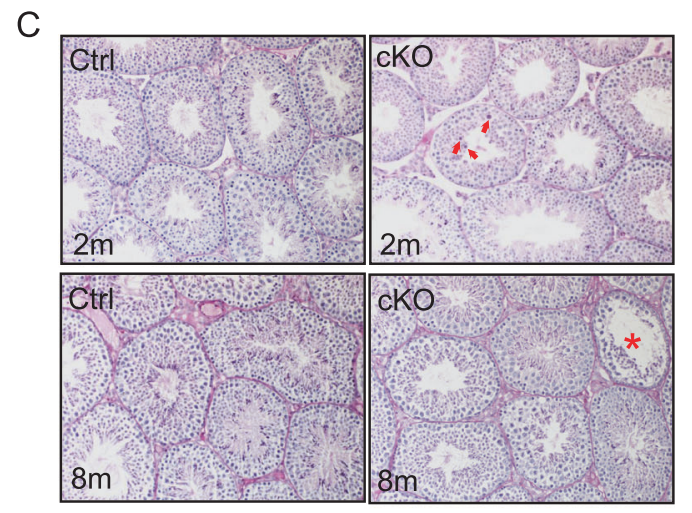

E

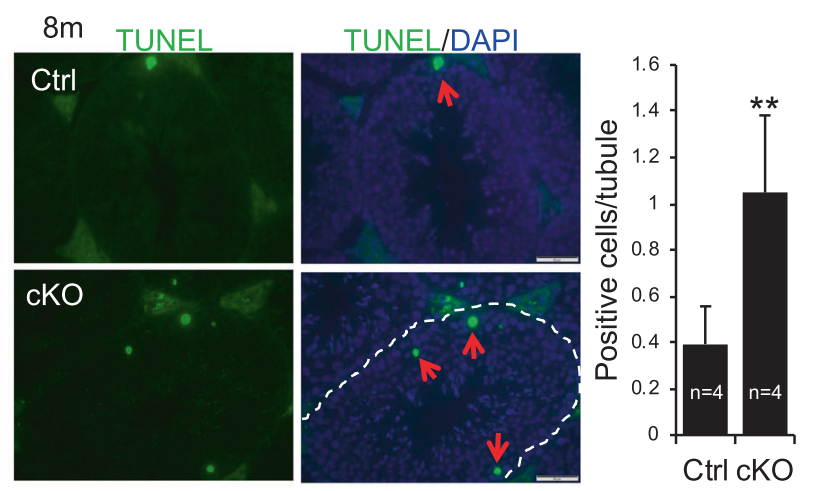

of vacuole-containing tubules (indicated by asterisks) to the total number of tubules are presented (right panel). 219-272 tubules per testis were examined. Tubules with complete depletion of spermatocytes in L3mbtl2 cKO testis are indicated by \#. e Representative images and quantification of TUNEL- positive cells in control and L3mbtl 2 cKO testes at 8 months of age. Red arrows indicate TUNEL positive cells in tubules. 168-279 tubules per testis were counted for apoptotic cells. The numbers of mice are indicated. In panels $\mathbf{a}$ and $\mathbf{b}$, */**/*** indicate comparisons between Ctrl and cKO; \#\#/\#\#\# indicate comparisons between 8 or 13 months with 2 months; $\$$ indicate comparisons between 8 and 13 months. $* p<0.05$; **p $p<0.01$; *** $p<$ $0.001 ; \# \# p<0.05 ; \# \# p<0.001 ; \$ \$ p<0.01$

We and others have shown that L3MBTL2 functions as a transcriptional repressor as it is an integral component of atypical polycomb repressive complex 1 (PRC1)-family complexes containing E2F6, RING2, HP1 $\gamma$, and MBLR in a number of cancer cell lines [19, 22] and in mouse embryonic stem (ES) cells [16]. Several PcG proteins including BMI1 and RING2 have been found to be recruited to DNA damage sites and participate in DNA damage response [23-28]. Interestingly, our previous study demonstrated that L3MBTL2 reduced cisplatin-induced DNA damage in renal tubular epithelial cells [29].

Two MBT domain-containing proteins SCMH1 and SCML2 have been found to be critically involved in spermatogenesis [30-32]. L3mbtl2 mRNA levels in the testis are the highest of all tissues analyzed [16]. In this study, we first examined cellular localization of L3MBTL2 in mouse 
testis, and then generated a mouse line with L3mbtl2 specifically deleted in spermatocytes (L3mbtl2 cKO). L3mbtl 2 cKO male mice exhibited premature aging in the testicular function, which was associated with defects in DNA damage and synapsis during meiosis I, and in histone acetylation, protamine deposition and chromatin condensation during spermiogenesis.

\section{Results}

\section{L3MBTL2 is mainly expressed in pachytene spermatocytes in mice}

As shown by immunohistochemistry (Supplemental Fig. S1A), L3MBTL2 was expressed in meiotic spermatocytes, with the highest expression found in pachytene spermatocytes. Less amounts of L3MBTL2 were expressed in spermatids.

During the first wave of spermatogenesis in mice, pachytene spermatocytes are the most advanced cell type present in the seminiferous tubules at 14 days of age. Interestingly, L3MBTL2 protein increased from 6 to 14 dpp, followed by slight decreases at $20 \mathrm{dpp}$ (Supplemental Fig. S1B). These results confirm the higher L3MBTL2 expression in pachytene spermatocytes.

\section{Generation of germ cell-specific L3mbtl2 cKO mice}

Floxed L3mbtl2 male mice (L3mbtl $2^{\mathrm{f} / \mathrm{f}}$ ) [29] were crossbred with Stra8-icre female mice to generate L3mbtl 2 conditional knockout mice (cKO). L3mbtl2 mRNA and protein levels were reduced by 98 and $87 \%$ respectively in the L3mbtl 2 cKO testes compared to $\mathrm{L} 3 \mathrm{mbtl} 2^{\mathrm{f} / \mathrm{f}}$ (control) testes (Supplemental Fig. S2A, S2B). Immunohistochemistry confirmed the drastic reduction in L3MBTL2 expression in cKO testes (Supplemental Fig. S2C). Genotyping PCR of the offspring born from L3mbtl2 $\mathrm{cKO}$ male mice mated with WT female mice showed no floxed allele in the offspring, suggesting that all the floxed alleles in the germ cells of paternal cKO mice were excised (Supplemental Fig. S2D).

\section{Deletion of L3mbtl2 in testis causes reduced testis size, sperm count, and fertility}

L3mbtl 2 cKO mice did not differ in body weight from WT controls (data not shown). However, they exhibited decreases in testis weight as compared with WT controls at 2, 8, and 13 months of age (Fig. 1a). The numbers of sperm in the caudal epididymis were similar between L3mbtl2 cKO and WT mice at 2 months of age, but were decreased in L3mbtl 2 cKO mice compared to WT mice at 8 and
13 months of age (Fig. 1b). The ratio of morphologically normal sperm was lower in L3mbtl 2 cKO mice than in WT mice (Supplemental Fig. S3B). The abnormal sperm in L3mbtl2 cKO mice showed defective head, and hairpin or $\mathrm{V}$-shape bending at the neck, the annulus between midpiece and principal piece, and the junction between principal piece and end piece (Supplemental Fig. S3A).

We then examined the fertility of male L3mbtl $2 \mathrm{cKO}$ mice (Supplemental Fig. S4). We paired 6-week-old control or L3mbtl2 cKO male mice with 6-week-old WT female mice. During the initial weeks, the litter sizes and litter intervals were similar between control and cKO mice. L3mbtl 2 cKO mice ceased to produce progeny at around 30 weeks of age, while control males continued to sire pups until 48 weeks. These results suggest that loss of L3mbtl2 results in premature testicular dysfunction.

\section{Deletion of $\mathrm{L3mbt} / 2$ leads to spermatogenic defects}

To determine the underlying mechanisms responsible for the reduced fertility, we first examined the histological structure of the testes from L $3 \mathrm{mbtl} 2 \mathrm{cKO}$ mice. L3mbtl2 cKO mice at 2 and 8 months of age exhibited a full spectrum of spermatogenic cells, with a small number of tubules showing giant multinucleated cells (MNCs) and germ cell loss (Fig. 1c). At 13 months of age, much more vacuolescontaining seminiferous tubules were found in L3mbtl2 cKO than in control testes (Fig. 1d). Some mutant tubules were depleted of the entire layer of spermatocytes, whereas many other mutant tubules lost all the germ cells (Fig. 1d). These results indicate that loss of $\mathrm{L} 3 \mathrm{mbtl} 2$ progressively impairs spermatogenesis.

There was no difference in mRNA levels of the testosterone responsive transcript Rhox 5 between the two genotypes (Supplemental Fig. S5), thus indicating that the mutant mice had normal testosterone levels.

TUNEL-positive germ cells were increased in L3mbtl2 cKO compared to WT mice at 8 months of age (Fig. 1e), perhaps explaining the germ cell loss in $\mathrm{L} 3 \mathrm{mbtl} 2 \mathrm{cKO}$ mice. Our previous study showed that L3MBTL2 negatively regulates p21 [16]. Interestingly, L3MBTL2 protein declined in WT testis from 28 days to 8 months of age, which was associated with increases in p21 expression (Supplemental Fig. S6A). L3mbtl2 deletion further increased p21 expression in the testes of 8-month-old mice (Supplemental Fig. S6B). These results implicate L3MBTL2 as an anti-testicular aging protein.

\section{Gene expression profiles}

Previous reports have demonstrated that L3MBTL2 represses transcription in vitro [16, 19, 22]. In order to gain insight into the global gene expression changes 


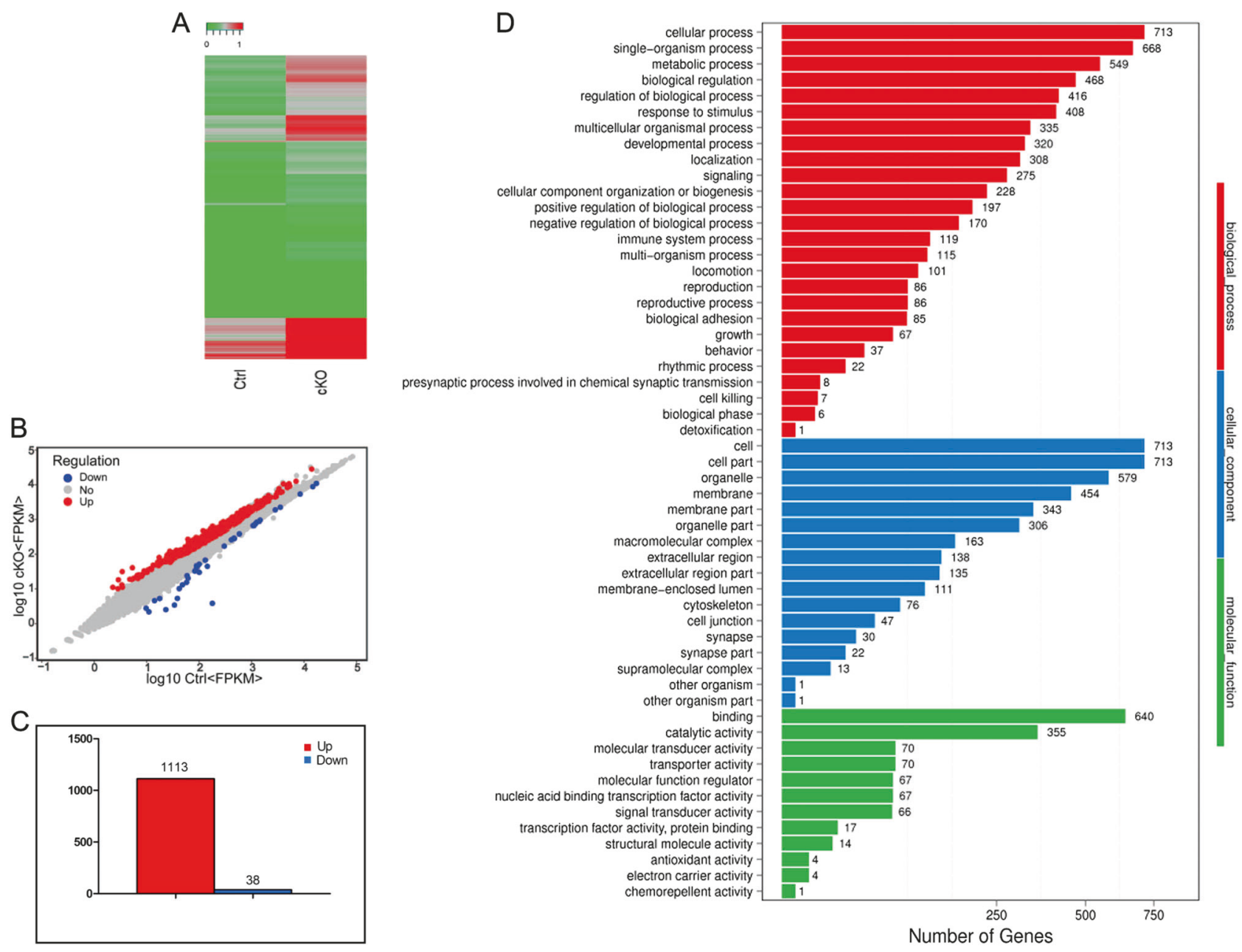

Fig. 2 RNA-sequencing in control and L3mbtl2 cKO testes identified differentially expressed genes. a Heat map of 1,151 genes that are differentially expressed between control (Ctrl) and L3mbtl2 cKO testes. These differentially expressed genes were chosen by $\geq 1.5$-fold change and $q$-value $<0.05$. Green to red $(0-1)$ represents the gradient increase of expression levels. b Comparison of gene expression between control ( $x$ axis) and L3mbtl 2 cKO testis ( $y$ axis). Red dots indicate upregulation, and blue dots indicate downregulation. c Bar chart of the numbers of differently expressed genes in L3mbtl $2 \mathrm{cKO}$ testis. 1113 genes (red) show upregulation, and 38 genes (blue) show downregulation. d The top GO terms associated with biological process, cellular component, and molecular function of differently expressed genes are shown. $(n=3)$

mainly related to binding and catalytic activity (Fig. 2d, green bars). KEGG analysis showed that the most enriched pathways included metabolic pathway, cancer and PI3KAKT signaling (Supplemental Fig. S7). However, only a few genes for steroid biosynthesis, which is related to spermatogenesis, were found to be differentially regulated (Supplemental Fig. S7).

Our previous study identified a panel of spermatogenesis genes including Ddx4, Tex19, Piwil2, Stk31, Tcam1, Hoxb8, Sox17, and Foxa2, which are bound and suppressed by L3MBTL2 in ES cells [16]. Surprisingly, these genes were not increased in $\mathrm{L} 3 \mathrm{mbt} 2 \mathrm{cKO}$ testes compared to WT testes as shown by the RNA-Seq. Furthermore, our realtime PCR analysis showed that Sox 17 was even decreased in L3mbtl2 cKO testes whereas other genes were not altered (Supplemental Fig. S8). The data indicate that while, the GO terms enriched for molecular function were 
A
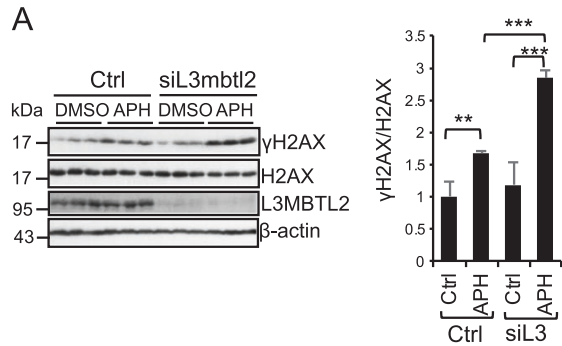

D

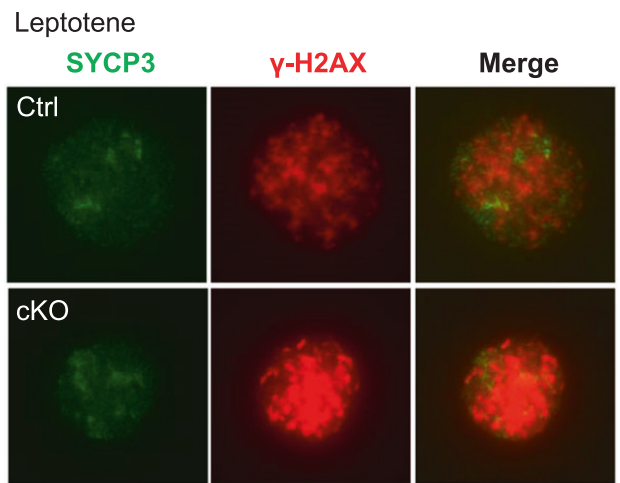

$8 \mathrm{~m}$

Fig. 3 L3mbtl2 deficiency induces DNA damage and defective $\gamma \mathrm{H} 2 \mathrm{AX}$ accumulation and removal during meiotic prophase. a-c Effects of L3MBTL2 knockdown on $\gamma \mathrm{H} 2 \mathrm{AX}$ expression in Hela cells in the presence of $\mathrm{APH}$, Zeocin, or $\gamma$-irradiation. Hela cells were transfected with control or L3mbt12 siRNAs (siL3mbt12 or siL3). Cells were treated with $0.5 \mu \mathrm{M}$ APH for $24 \mathrm{~h}$ (a), $100 \mu \mathrm{g} / \mathrm{ml}$ Zeocin for $1 \mathrm{~h}$ (b), or 5 Gy $\gamma$-irradiation (c), before the cells were harvested for Western blotting for $\gamma \mathrm{H} 2 \mathrm{AX}, \mathrm{H} 2 \mathrm{AX}$, and L3MBTL2. $\gamma \mathrm{H} 2 \mathrm{AX}$ levels relative to $\mathrm{H} 2 \mathrm{AX}$ levels were quantified by densitometry as indicated. $\mathbf{d} \gamma \mathrm{H} 2 \mathrm{AX}$ staining in control (L3mbtl $2^{\mathrm{f} / \mathrm{f}}$ ) and $\mathrm{L} 3 \mathrm{mbtl} 2 \mathrm{cKO}$ (L3mbtl2/f/f-Stra8-icre) primary spermatocyte nuclei at leptotene of meiotic prophase. SYCP3 accumulates on chromosomes beginning in

spermatogenesis associated genes were not appreciably affected by L3mbtl2 disruption in testis.

\section{Deletion of $\mathrm{L} 3 \mathrm{mbt} / 2$ leads to defective meiosis}

We recently revealed that L3MBTL2 inhibits the initial DNA damage induced by cisplatin by compacting chromatin in renal tubular epithelial cells [29]. To extend this finding, we treated Hela cells with the replication stress inducer aphidicolin (APH), which largely induces singlestrand breaks. APH treatment for $24 \mathrm{~h}$ induced $\gamma \mathrm{H} 2 \mathrm{AX}$ expression. Inhibition of L3mbtl2 further increased $\gamma \mathrm{H} 2 \mathrm{AX}$ expression (Fig. 3a). Inhibition of L3MBTL2 also increased $\gamma \mathrm{H} 2 \mathrm{AX}$ expression induced by Zeocin ( $1 \mathrm{~h})$ or $\gamma$-irradiation (5 Gy), which triggers DSB (Fig. 3b, c). These results indicate that L3MBTL2 inhibits DNA breaks induced by a variety of stimuli.

Mammalian meiosis involves extensive DNA damage. DSBs are generated during prophase of meiosis I. $\gamma \mathrm{H} 2 \mathrm{AX}$ accumulates in the nuclei of leptotene and zygotene

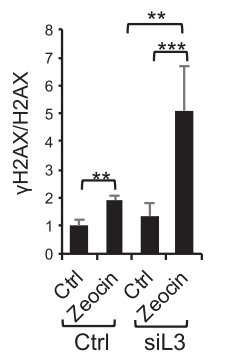

E

Pachytene

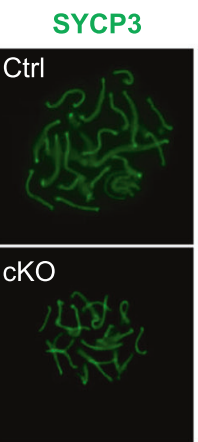

C

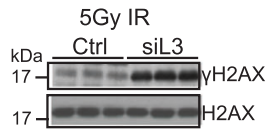

F

Abnormal $\mathrm{YH} 2 \mathrm{AX}$ staining

in pachytene

nuclei
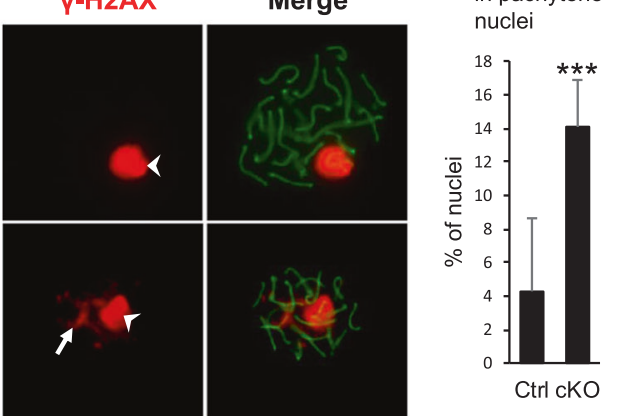
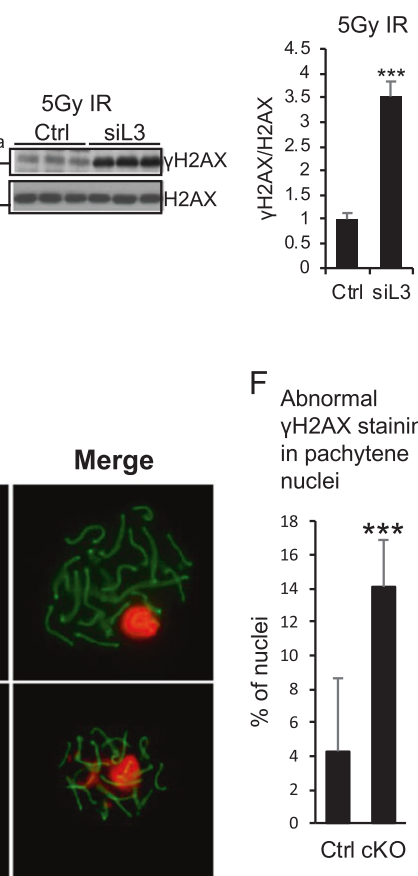

leptotene. $\gamma \mathrm{H} 2 \mathrm{AX}$ signals were quantified by ImageJ (right panel). More $\gamma \mathrm{H} 2 \mathrm{AX}$ is accumulated in the nuclei in L3mbtl2 cKO than in control mice. 3 mice were used for each group. e $\gamma \mathrm{H} 2 \mathrm{AX}$ staining in control and L3mbtl $2 \mathrm{cKO}$ primary spermatocyte nuclei at pachytene stages of meiotic prophase. SYCP3 is present along their full length during pachytene stage. $\gamma \mathrm{H} 2 \mathrm{AX}$ is restricted to the $\mathrm{XY}$ body (white arrow head) in control spermatocytes, but remains at some autosomal locations (white arrow) in L3mbtl 2 cKO spermatocytes. f Percentage of pachytene spermatocytes retaining $\gamma \mathrm{H} 2 \mathrm{AX}$ in the autosomal region in control and $\mathrm{L} 3 \mathrm{mbtl} 2 \mathrm{cKO}$ mice. 3 mice were used for each group. $* p<0.05 ; * * p<0.01 ; * * * p<0.001$

spermatocytes, in which DSBs are formed, but it is removed from the chromosomes during the process of repairing, and is absent from the autosomes by the pachytene stage [33, 34]. We prepared meiotic spreads using control and L3mbtl2 cKO testes at 8 months of age, followed by immunofluorescence for $\gamma \mathrm{H} 2 \mathrm{AX}$ and the synaptonemal complex (SC) component SYCP3. $\gamma \mathrm{H} 2 \mathrm{AX}$ accumulated on chromosomes during the leptotene stage in control meiotic cells, and this deposition was much increased in cKO meiotic cells (Fig. 3d). As expected, $\gamma \mathrm{H} 2 \mathrm{AX}$ was removed from the autosomes during the pachytene stage in control testes. However, $\gamma \mathrm{H} 2 \mathrm{AX}$ was inappropriately retained on autosomes in pachytene spermatocytes (Fig. 3e; Supplemental Fig. S9) in L3mbtl2 cKO testes. These defects were evident in a subset of cells (Fig. 3f). These results suggest that L3mbtl2 deletion increases initial DNA damage load in leptotene spermatocytes, which results in incomplete resolution in pachytene spermatocytes.

DNA breaks are followed by invasion of the homologous chromosomes and crossing over, which involve recruitment 

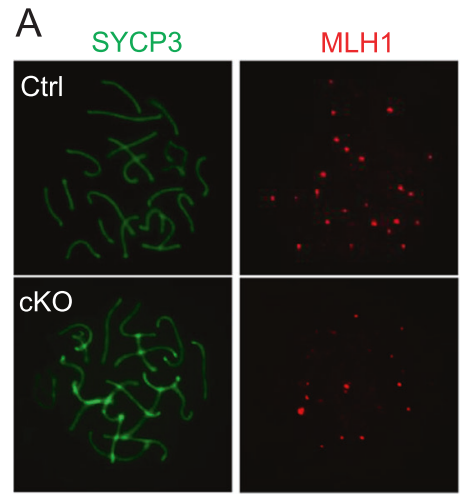

$8 m$
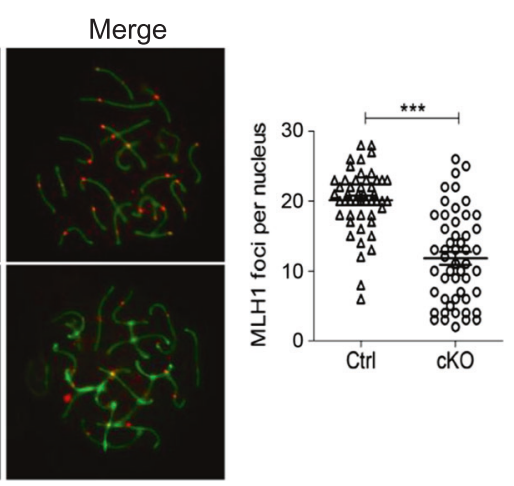

Fig. 4 L3mbt12 deficiency induces defects in crossing-over and synapsis in pachytene spermatocytes. a Costaining of MLH1 and the SC component SYCP3 shows reduced number of MLH1 foci in L3mbtl2 cKO pachytene spermatocytes. MLH1 foci per pachytene

of MLH1/MLH3 heterodimer during middle to late pachytene stages $[35,36]$. We found that $\mathrm{L} 3 \mathrm{mbtl} 2 \mathrm{cKO}$ pachytene spermatocytes exhibited reduced recruitment of MLH1 (Fig. 4a), indicating impaired crossover formation.

Failure to resolve DSBs and to form interhomolog crossovers is associated with defects in synapsis. We did not see any differences in SYCP3, a lateral element of the SC, which assembles on unpaired chromosomes during early prophase, between control and L3mbtl2 deficient spermatocytes. SYCP1, a central element of the SC, assembles only on synapsed chromosomes. Our results showed that whereas control pachytene nuclei had continuous SYCP1 staining along the chromosomes, many L3mbtl2 cKO pachytene spermatocytes had discontinuous SYCP1 staining (Fig. 4b), indicating incomplete SC formation. Taken together, resolution of DNA breaks, crossing-over and synapsis are incomplete in a subset of spermatocytes in L3mbt12 cKO testis. These meiotic defects may explain the increased apoptosis present in spermatocytes in L3mbt12 cKO testes.

Previous studies have shown that the MBT family members Scmh1 and Scml2 modulate chromatin modifications of the XY body including exclusion of H3K27me3 and enrichment of H3K9me1 and H3K4me2 [30-32, 37]. $\mathrm{We}$, therefore, examined these modifications in the meiotic spreads of L3mbtl2 cKO testes. Neither exclusion of H3K27me3 nor enrichment of H3K9me1 in the XY body was different between control and L3mbtl2 cKO pachytene spermatocytes (Supplemental Fig. S10A, S10B, S10D). In addition, H3K4me2 enrichment in pachytene (data not shown) and diplotene (Supplemental Fig. S10C, S10D) was not altered either by L3mbt12 deletion. 53BP1 expression in the XY body was similar between control and L3mbtl 2 cKO testes (Supplemental Fig. S11). These results suggest that L3mbtl2 may not regulate the XY chromatin modifications and repair.
B
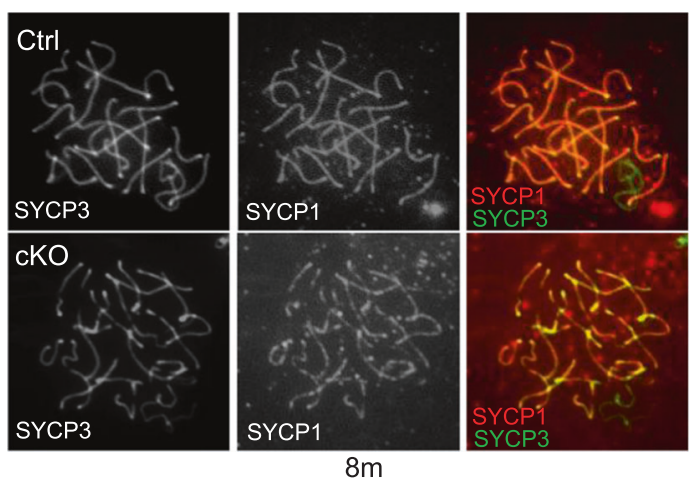

nucleus in control and L3mbt12 cKO are presented. $* * * p<0.001$. b Costaining of lateral (SYCP3) and central (SYCP1) elements of the SC shows discontinuous SYCP1 staining in L3mbtl2 cKO pachytene spermatocytes, indicating defective SC formation

\section{Deletion of L3mbt|2 alters histone modifications in germ cells and disrupts protamine 1 deposition in sperm}

To determine whether acetylation of core histones is altered in $\mathrm{L} 3 \mathrm{mbtl} 2 \mathrm{cKO}$ mice, we examined acid-extracted proteins from the chromatin of germ cell fractions. We found that acetylation levels of H2B at K16 (H2BAck16), H3 at K18 (H3AcK18) and $\mathrm{H} 4$ at K5, K8, K12, and K16 (H4tetraAcK) were not significantly altered in L3mbtl2 cKO mice as compared with control mice at 2 months of age (Fig. 5a). Strikingly, these acetatylation levels in germ cells were reduced in $\mathrm{L} 3 \mathrm{mbtl} 2 \mathrm{cKO}$ mice compared to control mice at 8 months of age (Fig. 5b). Immunofluorescence on stage $\mathrm{X}$ tubules, the stage at which round spermatids start to elongate, showed that H4tetraAcK was localized to elongating spermatids in control testes, and this expression was dramatically reduced in cKO testes (Fig. 5c). H2BAcK16 levels in elongating spermatids were also much reduced in cKO testes (Fig. 5d).

We then examined whether L3mbtl2 cKO mice exhibit defects in the histone to protamine transition. The transition proteins BRDT, TNP1, and TNP2 and protamines 1 and 2 did not show differential expression at mRNA levels between control and L3mbt12 cKO mice (Supplemental Fig. S12). However, protamine 1 deposition in sperm isolated from caudal epididymides was much reduced in $\mathrm{L} 3 \mathrm{mbtl} 2 \mathrm{cKO}$ mice compared to control mice (Fig. 6a). Immunofluorescence on caudal epididymides also revealed a dramatic reduction in protamine 1 signal in L3mbtl2 cKO mice (Fig. 6b).

Chromatin condensation is the event that succeeds the histone to protamine transition. Therefore, we examined the extent of chromatin condensation during the transformation of round spermatids into elongated spermatids in the testis using transmission electron microscopy (Fig. 5e, f). Round spermatids at steps 5-7 were similar in structure between 
A

\section{Germ cells $(2 \mathrm{~m})$}

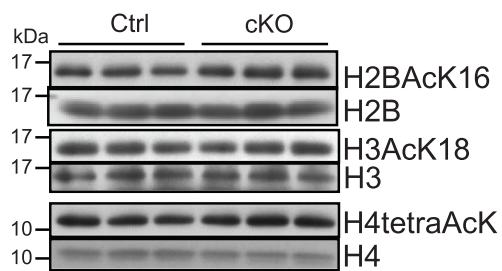

B Germ cells $(8 \mathrm{~m})$

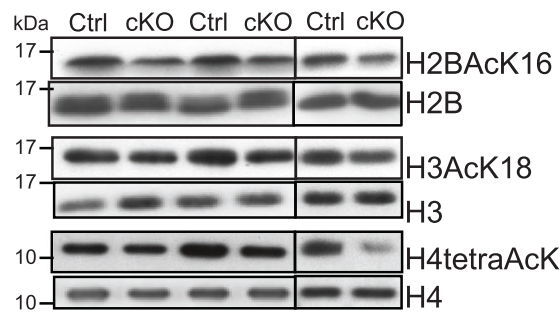

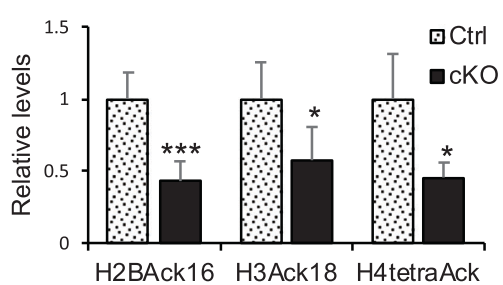

C
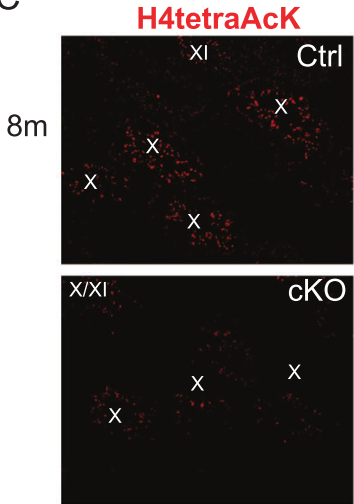

Merge

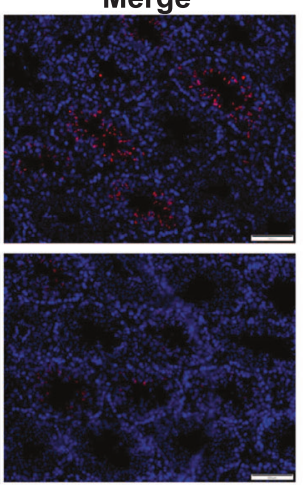

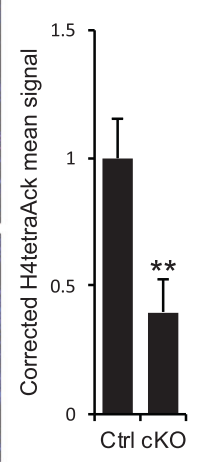
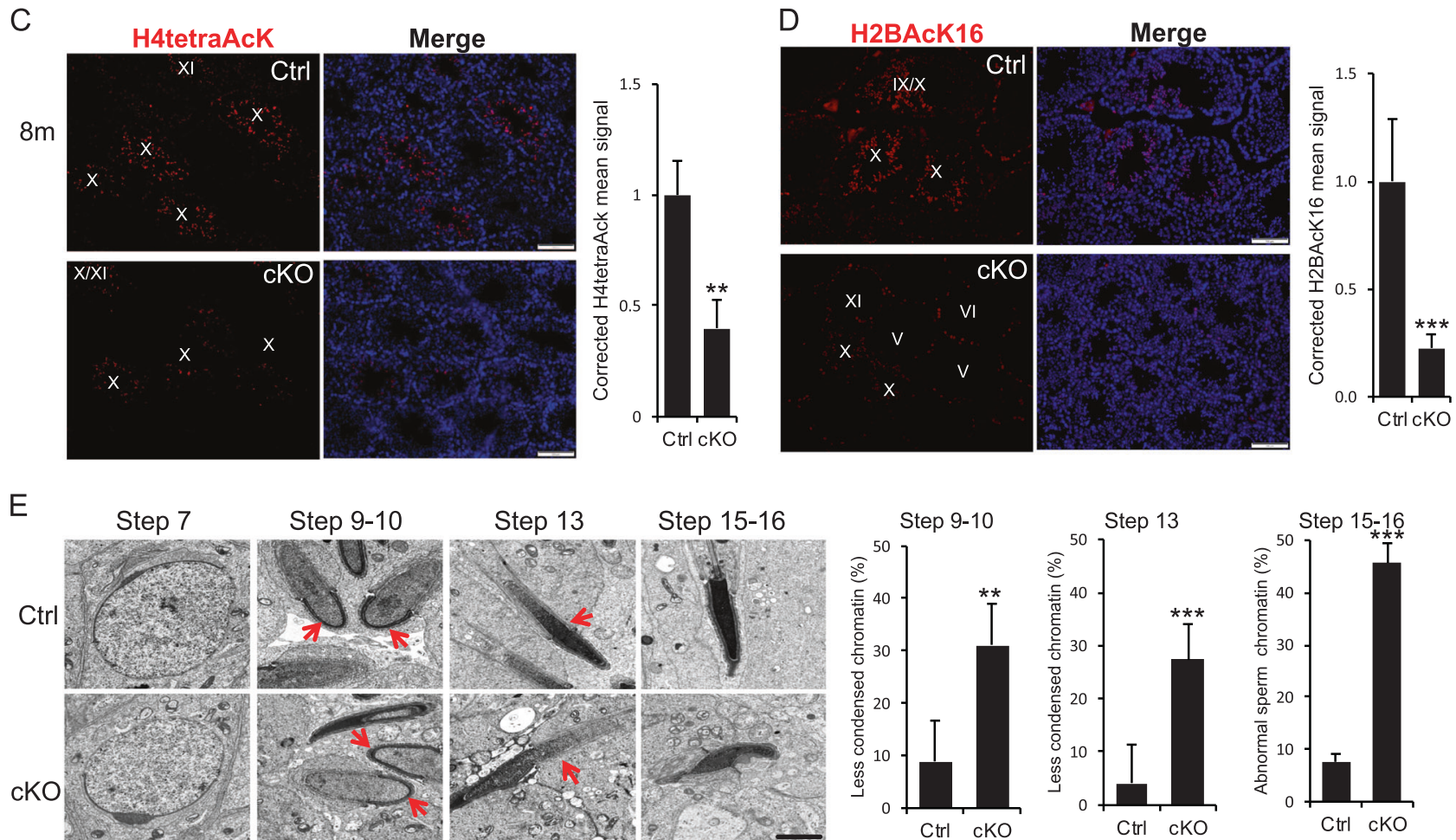

Step $9-10$

Step 13

Step 15-16
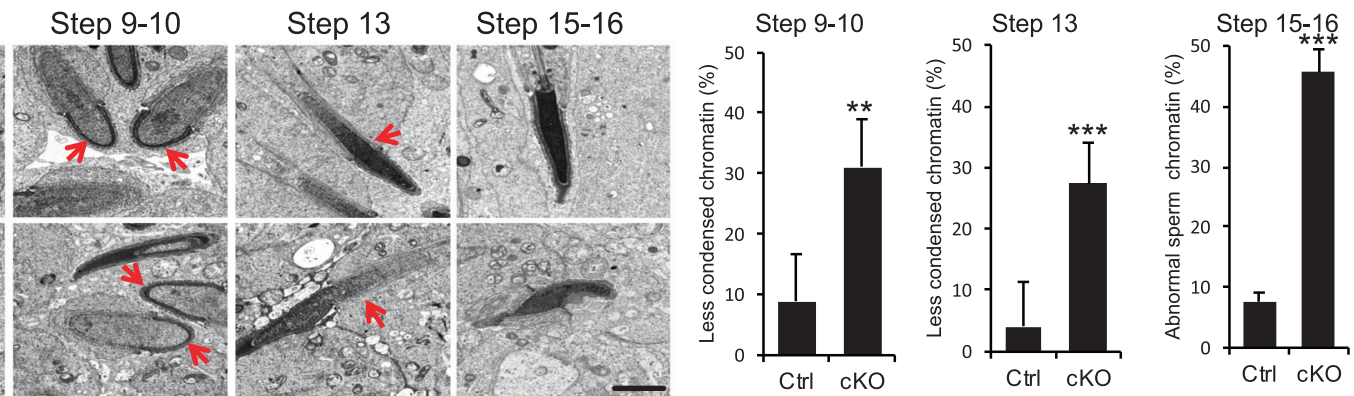

Fig. 5 Histone acetylation in the germ cells of L3mbtl $2 \mathrm{cKO}$ mice. a Western blot analyses of H2BAcK16, H3AcK18, and H4tetraAcK on acid extracts of germ cell chromatin from control (L3mbtl $2^{\mathrm{f} / \mathrm{f}}$ ) and L3mbtl $2 \mathrm{cKO}$ (L3mbt12 ${ }^{\mathrm{f} / \mathrm{f}}$-Stra8-icre) mice at 2 months of age. b Western blot analyses of H2BAcK16, H3AcK18, and H4tetraAcK on acid extracts of germ cell chromatin from three littermate pairs of control and L3mbtl $2 \mathrm{cKO}$ mice at 8 months of age (right panel). H2BAcK16 levels relative to $\mathrm{H} 2 \mathrm{~B}$ levels, $\mathrm{H} 3 \mathrm{AcK} 18$ levels relative to $\mathrm{H} 3$ levels, and $\mathrm{H} 4$ tetraAcK levels relative to $\mathrm{H} 4$ levels were quantified by densitometry (right panel). c, d Immunofluorescence for H4tetraAcK and H2BAcK16 in stage $\mathrm{X}$ seminiferous tubules from 8-month-old control and L3mbtl2 cKO mice. H4tetraAcK and H2BAcK16 signals in spermatids at stage $\mathrm{X}$

were quantified by ImageJ. 3 mice were used for each group. e Defective DNA condensation during steps 9-16 of spermatid development in L3mbtl 2 cKO testes. Typical electron microscopic images of spermatids at different steps are shown. No difference was found in round spermatids (Step 7) between L3mbtl 2 cKO and control testes. Decreased density was found in spermatids at step 9-10 (red arrows), when histoneprotamine exchange starts, in L3mbtl 2 cKO mice. Step 13 spermatids show anterior-to-posterior thickening of chromatin fibers (DNA condensation, red arrows). f Frequency of spermatids with less condensed chromatin at steps $9-10$ and 13 and with abnormal chromatin at steps $15-16$ was summarized. 3 mice were used for each genotype. $* p<0.05$; $* * p<0.01 ; * * * p<0.001$

control and L3mbtl2 cKO mice. Elongating spermatids at steps 9-10 and condensing spermatids at step 13, showed reduced density of nuclei in L3mbtl2 cKO testes as compared to control testes. In condensed spermatids at step 1516, nuclear morphology and chromatin compaction of spermatid heads were severely disrupted in L $3 \mathrm{mbtl} 2 \mathrm{cKO}$ mice. In epididymides, sperm heads were less compacted and residual cytoplasm were not completely removed in most of the sperm in L3mblt2 cKO mice (Fig. 6c). These results suggest that L3mbtl2 deficiency results in reduced histone acetylation, protamine deposition and chromatin condensation during spermiogenesis.

\section{Deletion of $\mathrm{L} 3 \mathrm{mbt} \mid 2$ reduces RNF8 and ubH2A levels in elongating spermatids}

RNF8 is an E3 ubiquitin ligase, and it directly ubiquitinates $\mathrm{H} 2 \mathrm{~A}$ and $\mathrm{H} 2 \mathrm{~B}[34,38]$. Ubiquitinated $\mathrm{H} 2 \mathrm{~A}(\mathrm{ubH} 2 \mathrm{~A})$ and 


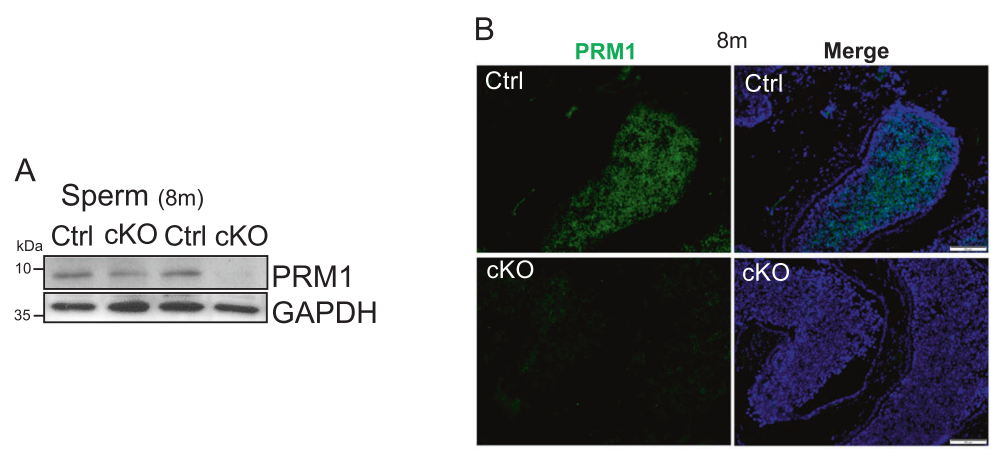

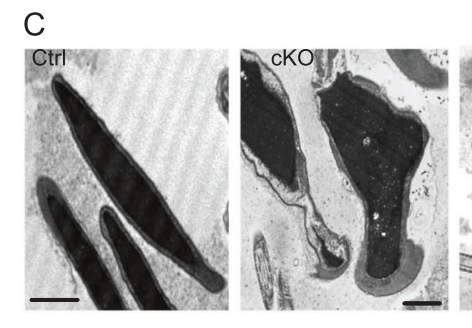

Fig. $6 \mathrm{~L} 3 \mathrm{mbtl} 2$ deficiency leads to reduced protamine 1 deposition in sperm. a Immunoblots for protamine 1 (PRM1) in sperm lysates from the caudal epididymides of control $\left(\mathrm{L} 3 \mathrm{mbtl} 2^{\mathrm{f} / \mathrm{f}}\right.$ ) and $\mathrm{L} 3 \mathrm{mbtl} 2 \mathrm{cKO}$ (L3mbtl $2^{\mathrm{f} / \mathrm{f}}$-Stra8-icre) at 8 months of age. b Immunofluorescence for PRM1 on caudal epididymal cross sections from control and L3mbtl2

H2B (ubH2B) control H4 acetylation through a transhistone modification mechanism $[13,14]$. To understand the molecular basis for the impaired histone-to-protamine exchange during spermiogenesis in L3mbtl2 cKO mice, we studied the RNF8-ubH2A/ubH2B pathway. First, we tested whether L3MBTL2 physically interacts with RNF8. Indeed, we found that HA-RNF8 and L3MBTL2-Flag were able to pull down each other in co-transfected HEK293T cells (Fig. 7a, b). In the testis, L3MBTL2 also interacted with RNF8 (Fig. 7c). The interaction of L3MBTL2 with RNF8 recently was also demonstrated by Nowsheen and colleagues [39] during the submissions of our manuscript. Strikingly, nuclear RNF8 protein levels in germ cells were much reduced in L3mbtl2 cKO compared to control mice (Fig. 7f), whereas total RNF8 mRNA (Fig. 7d) and protein (Fig. 7e) levels did not change between the two genotypes. Immunohistochemistry showed that RNF8 in the nucleus of elongating spermatids were reduced by $\mathrm{L} 3 \mathrm{mbtl} 2$ deletion (Fig. $7 \mathrm{~g}$ ). In acid-extracted proteins from the chromatin of germ cell fractions, ubH2A levels were also much lower in $\mathrm{L} 3 \mathrm{mbtl} 2 \mathrm{cKO}$ than in control testes (Fig. 7h), while ubH2B did not change (data not shown). As shown by immunohistochemistry, ubH2A signals in elongating spermatids were weaker in L3mbtl2 cKO than in control mice, while the signals in other cell types were similar between the two genotypes (Fig. 7i).

To consolidate the observations from the testis, we studied the RNF8/ubH2A pathway in GC2 spermatocyte cell line. Inhibition of L3MBTL2 did not alter RNF8 mRNA
cKO mice at 8 months of age. Scale bar: $200 \mu \mathrm{m}$. c Sperm in the caudal epididymides of L3mbt12 cKO mice were less condensed and contained residual cytoplasm. Typical electron microscopic images of sperm heads from control (Ctrl) and L $3 \mathrm{mbtl} 2 \mathrm{cKO}$ mice at 8 months of age. 3 mice were used for each genotype for panels $\mathbf{b}$ and $\mathbf{c}$

(Fig. 8a) and protein (Fig. 8b) expression, but drastically reduced nuclear RNF8 levels (Fig. 8c). ubH2A levels were also reduced by L3MBTL2 knockdown (Fig. 8d). These results indicate that $\mathrm{L} 3 \mathrm{MBTL} 2$ and RNF8 form a complex, and deletion of L3mbtl2 suppresses the RNF8-ubH2A pathway in the elongating spermatids.

\section{Discussion}

In this study we show that deletion of L $3 \mathrm{mbtl} 2$ in male germ cells did not alter the fertility in young mice, but led to premature reproductive cessation in aging mice. We observed defects in $\gamma \mathrm{H} 2 \mathrm{AX}$ deposition and removal, synapsis and crossing over during prophase of meiosis I in the testes of $\mathrm{L} 3 \mathrm{mbtl} 2 \mathrm{cKO}$ mice at 8 months of age. We also found decreased activity of the RNF8-ubH2A pathway and the subsequent histone acetylation in spermatids and decreased protamine deposition and chromatin condensation in sperm in the mutant mice. These defects in meiosis and spermiogenesis were associated with increased germ cell loss and abnormal spermatozoa in aging mice. Our observations suggest that L3mbtl2 deficiency causes premature testicular failure. In addition, mice lacking L3mbtl 2 in male germ cells showed smaller testes even at young ages. Therefore, L3MBTL2 also plays a role in testis development.

Meiotic spermatocytes contained the highest levels of L3mbtl2. Deletion of L3mbtl2 led to more apoptosis and 
A

\section{HEK293T cells}

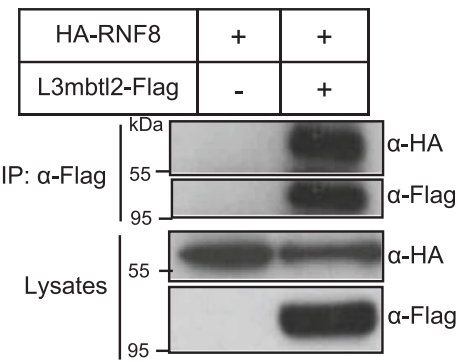

B HEK293T cells

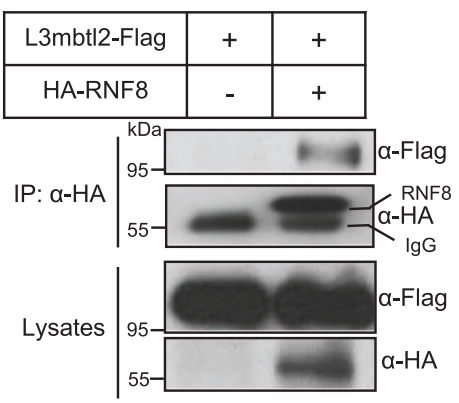

C

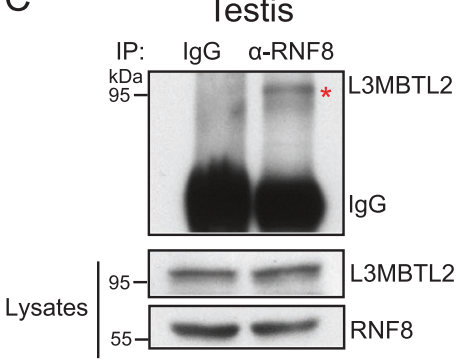

D

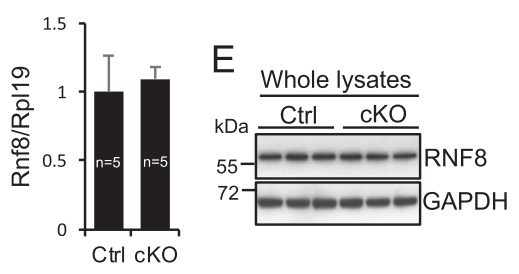

$\mathrm{H}$

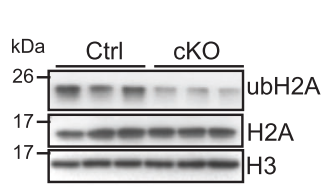

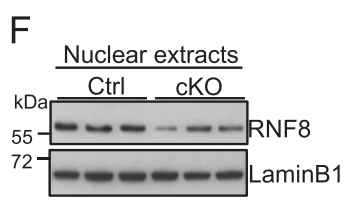

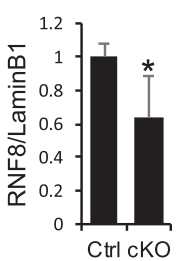

G

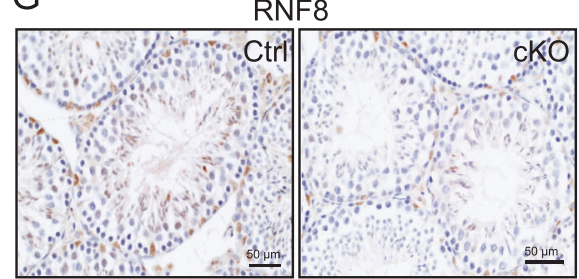

I

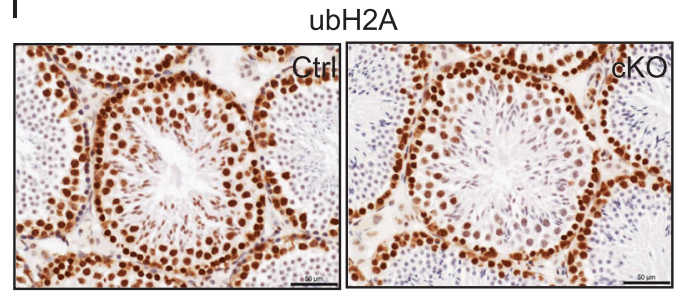

Fig. 7 L3MBTL2 interacts with RNF8 and L3mbtl2 deficiency leads to reduced nuclear RNF8 and ubH2A levels in elongating spermatids. a-c L3MBTL2 interacts with RNF8. HEK293 cells were transfected with HA-RNF8 in the absence or presence of L3mbtl2-Flag. Cell lysates were used for immunoprecipitation (IP) and subsequent Western blotting (WB) using indicated antibodies. Cell lysates were used for WB as indicated to show inputs (a). HEK293 cells were transfected with L3mbt12-Flag in the absence or presence of HA-RNF8. Cell lysates were used for IP and /or WB as indicated (b). Lysates from mouse testes were immunoprecipitated with anti-RNF8 antibody or normal $\mathrm{IgG}$, and the precipitates and lysates were subjected to WB as indicated (c). d mRNA levels of Rnf8 in germ cells from control and L3mbtl 2 cKO mice at 8 months of age. Rpl19 is the internal control. e Western blot analyses of RNF8 on whole lysates of germ cells from control and L3mbtl2 cKO mice at 8 months of age. GAPDH is the loading control. f Western blot analyses of RNF8 on nuclear extracts

even depletion of spermatocytes in some seminiferous tubules in aging testes. These data implicate an important role for L3mbtl2 in meiosis. Our previous study has shown that L3MBTL2 increases intital DNA damage load induced by cisplatin or $\gamma$-irradiation by compacting chromatin [29]. Consistently, we observed an increase in $\gamma \mathrm{H} 2 \mathrm{AX}$ signal on chromosomes during leptotene in L3mbtl2 cKO testes, indicating an abnormal initiation of meiotic recombination. The increased initial $\gamma \mathrm{H} 2 \mathrm{AX}$ signal may explain the retention of $\gamma \mathrm{H} 2 \mathrm{AX}$ on autosomes during pachytene stage. Defects in DSB and its repair are often associated with abnormal synapsis and crossing over [40]. Indeed, we found of germ cells from control and L3mbt12 cKO mice at 8 months of age (left panel). Nuclear RNF8 levels relative to LaminB1 levels were quantified by densitometry (right panel). g Immunohistochemical staining for RNF8 in the testes of control and L3mbtl2 cKO mice at 8 months of age. The signals for RNF8 in elongating spermatids were reduced in L3mbtl 2 cKO compared with control testes. $\mathbf{h}$ Western blot analyses of ubH2A on acid extracts of germ cell chromatin from control and $\mathrm{L} 3 \mathrm{mbtl} 2 \mathrm{cKO}$ mice at 8 months of age. ubH2A levels relative to $\mathrm{H} 2 \mathrm{~A}$ levels, and $\mathrm{H} 2 \mathrm{~A}$ levels relative to $\mathrm{H} 3$ levels were quantified by densitometry (right panels). i Immunohistochemical staining of ubH2A in the testes of control and L3mbtl $2 \mathrm{cKO}$ mice at 8 months of age. The signals for $\mathrm{ubH} 2 \mathrm{~A}$ in elongating spermatids were reduced in $\mathrm{L} 3 \mathrm{mbt} 2 \mathrm{cKO}$ compared with control testes while the signals in other cell types remained unchanged between the two genotypes

defective SC formation and meiotic crossover in L3mbtl2 cKO pachytene spermatocytes. Thus, our observations indicate that $\mathrm{L} 3 \mathrm{mbtl} 2$ is required for normal DSB, synapsis and homologous recombination during meiosis. The death of L3mbt12 mutant germ cells is probably attributed to the defective meiosis.

SCMH1, another MBT domain-containing protein, is most highly expressed in pachytene spermatocytes. SCMH1 is excluded from the XY body during the pachytene stage. Deletion of Scmh1 led to apoptotic elimination of late pachytene spermatocytes due to the failure in the exclusion of PRC1 proteins such as Phc1 and 

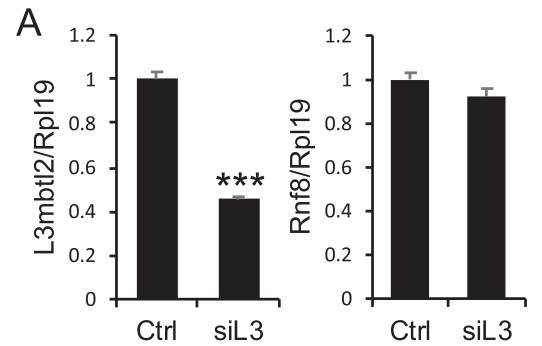

C

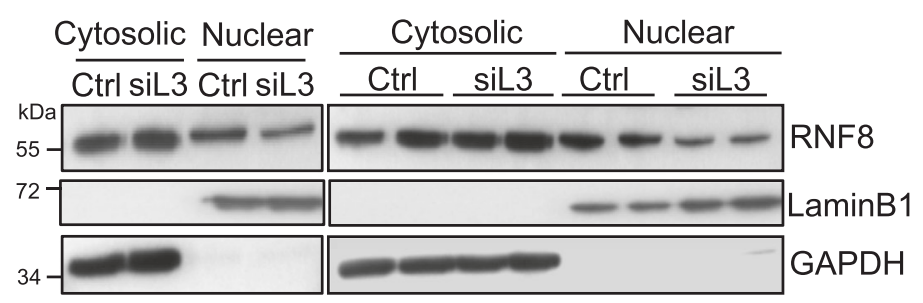

D

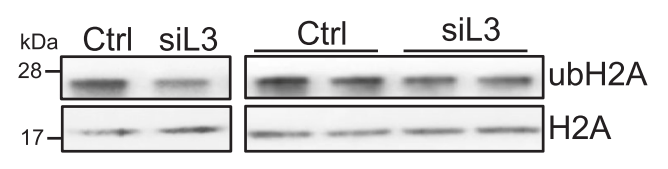

B
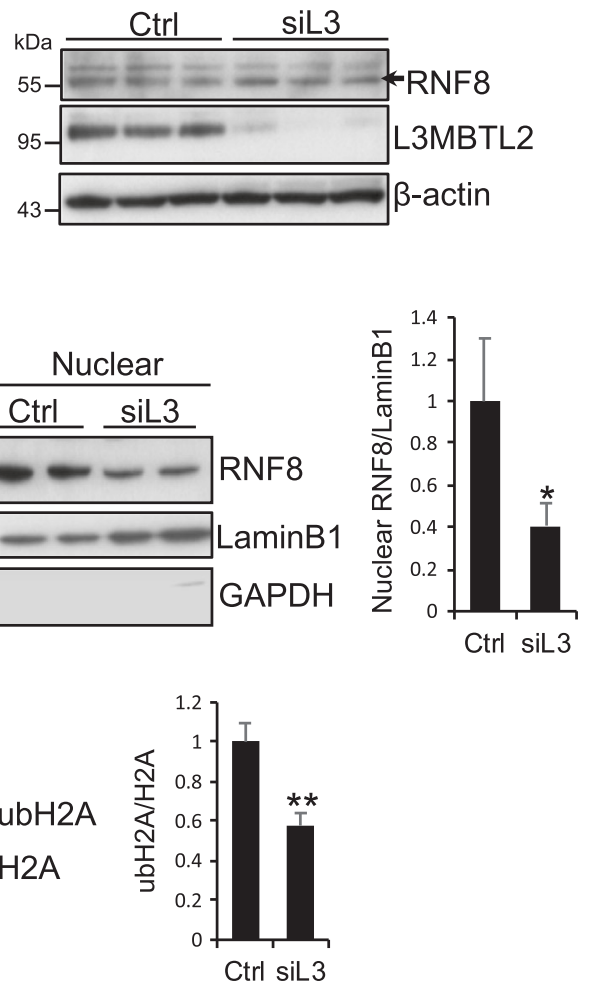

Fig. 8 Inhibition of L3MBTL2 reduces nuclear RNF8 expression and ubH2A levels in GC2 cells. a, b Effects of siRNA-mediated L3mbtl2 knockdown on Rnf8 mRNA and protein expression. GC2 cells were transfected with control (Ctrl) or L3mbtl2 siRNAs (siL3). $45 \mathrm{~h}$ after transfection, cells were serum-starved for $6 \mathrm{~h}$. Cells then were collected for real-time PCR analysis or Western blotting for L3mbtl 2 and Rnf8 mRNA (a) and protein (b) levels. c Effects of siRNA-mediated L3mbtl2 knockdown on nuclear RNF8 protein levels. GC2 cells were transfected with control (Ctrl) or L3mbtl2 siRNAs (siL3). $45 \mathrm{~h}$ after transfection, cells were serum-starved for $6 \mathrm{~h}$ before cells were collected for nuclear protein extraction and western blotting for RNF8

Phc2 from the XY body and in the chromatin modifications of the XY body including exclusion of $\mathrm{H} 3 \mathrm{~K} 27 \mathrm{me} 3$ and accumulation of $\mathrm{K} 3 \mathrm{~K} 9 \mathrm{me} 1$ and $\mathrm{H} 3 \mathrm{~K} 9 \mathrm{me} 2$ [30]. SCML2 is also most highly expressed in pachytene spermatocytes. But, in contrast to SCMH1, SCML2 concentrates on the $\mathrm{XY}$ body, where it interacts with the deubiquitinase USP7 to maintain the normal level of H2A ubiquitination in the $\mathrm{XY}$ chromatin [31, 32]. Interestingly, neither SCMH1 nor SCML2 appeared to be critically involved in the regulation of DSB, synapsis and homologous recombination of autosomes [30-32]. Like SCMH1 and SCML2, L3MBTL2 is most highly expressed in pachytene spermatocytes, but the subcellular localization of L3MBTL2 could not be determined due to lack of high quality L3MBTL2 antibodies for immunofluorescence. Nevertheless, we found that deletion of L3mbtl2 did not alter the XY body formation, exclusion (left panel). LaminB1 and GAPDH were used for loading controls for nuclear and cytosolic extracts respectively. Nuclear RNF8 levels relative to LaminB1 levels were quantified by densitometry (right panel). d Effects of siRNA-mediated L3mbtl2 knockdown on ubH2A levels. GC2 cells were transfected with control (Ctrl) or L3mbtl2 siRNAs (siL3). $45 \mathrm{~h}$ after transfection, cells were serumstarved for $6 \mathrm{~h}$ before histones were extracted using $\mathrm{HCl}(0.2 \mathrm{~N})$. Acid extracts were used for western blotting for ubH2A and H2A (left panel). ubH2A levels relative to $\mathrm{H} 2 \mathrm{~A}$ levels were quantified by densitometry (right panel). $* p<0.05 ; * * p<0.01 ; * * * p<0.001$

of $\mathrm{H} 3 \mathrm{~K} 27 \mathrm{me} 3$, and accumulation of $\mathrm{H} 3 \mathrm{~K} 9 \mathrm{me} 1$, $\mathrm{H} 3 \mathrm{~K} 4 \mathrm{me} 2$, and 53BP1in the XY body.

We also identified defects in spermiogenesis including reduced acetylation levels of histone H2BK16, H3K18, and $\mathrm{H} 4 \mathrm{~K} 5,8,12$, and 16 in elongating spermatids, and reduced promatine 1 deposition in sperm in L3mbtl2 $\mathrm{cKO}$ mice at ages of 8 months. The chromatin of spermatids and sperm was found to be less condensed in L3mbtl2 $\mathrm{cKO}$ mice. Therefore, L3mbtl2 also plays an important role in the histone to protamine transition and subsequent chromatin condensation in aging mice. Interestingly, histone acetylation was not altered by L3mbtl2 deletion in young mice at 2 months of age, further suggesting the an age-dependent role of $\mathrm{L} 3 \mathrm{mbt} 2$ in the testis.

Previous studies have shown that RNF8 ubiquitinates $\mathrm{H} 2 \mathrm{~A}$ and $\mathrm{H} 2 \mathrm{~B}$, and $\mathrm{H} 2 \mathrm{~A}$ and $\mathrm{H} 2 \mathrm{~B}$ ubiquitination controls H4K16 acetylation [14, 34, 38]. In the present study, we 
found that L3MBTL2 interacted with RNF8, and deletion of L3mbtl2 reduced nuclear RNF8 and $\mathrm{ubH} 2 \mathrm{~A}$ levels in the elongating spermatids. These results may explain the defective histone-to-protamine transition observed in L3mbtl 2 cKO mice. Of note, L3MBTL2 also interacts with Ring1B [16, 22], another E3 ubiquitin ligase that mediates $\mathrm{H} 2 \mathrm{~A}$ ubiquitination [41]. It is unclear whether and how the interactions of L3MBTL2 with Ring1B are involved in the regulation of $\mathrm{H} 2 \mathrm{~A}$ ubiquitination in spermatids.

Taken together, we have found that $\mathrm{L} 3 \mathrm{mbt} 2 \mathrm{deficiency}$ leads to increased DNA breaks and inappropriate retention of $\gamma \mathrm{H} 2 \mathrm{AX}$ on autosomes, and defective crossing-over and synapsis during the pachytene stage of meiosis I. L3mbtl2 deficiency also results in decreases in the levels of the RNF8-ubH2A pathway and histone acetylation in elongating spermatids, and in protamine 1 deposition and chromatin condensation in sperm. Thus, L3MBTL2 is required for normal chromatin remodeling during meiosis and spermiogenesis.

\section{Materials and methods}

\section{Mice}

Floxed L3mbt12 mice have been previously described $[16,29]$. Floxed L3mbtl 2 mice $\left(\mathrm{L} 3 \mathrm{mbtl} 2^{\mathrm{f} / \mathrm{f}}\right)$ were crossed to Stra8-icre mice to obtain L3mbtl2 ${ }^{\mathrm{f} / \mathrm{wt}}$ mice. L3mbtl2 ${ }^{\mathrm{f} / \mathrm{wt}}$; Stra8-icre female mice then were mated with L3mbtl $2^{\text {f/f }}$ males to generate male germ cell specific L3mbtl 2 knockout (L3mbtl2 f/f; Stra8-icre) mice (cKO). In the Stra8-icre transgenic line, cre is expressed in pre-meiotic germ cells [42]. Therefore, the cre expression pattern allows cremediated recombination of the floxed L $3 \mathrm{mbt} 2$ gene to take place before the highest L3mbtl2 expression.

All animal studies were approved by The Chinese University of Hong Kong Animal Experimentation Ethics Committee, and were conducted in accordance with The Chinese University of Hong Kong animal care regulations.

\section{Histology and immunostaining}

Testes were isolated from mice at 2, 8, and 13 months of age, and fixed in Bouin's solution (Sigma). Sections in thickness of $5 \mu \mathrm{m}$ were used for periodic acid Schiff (PAS) staining and immunohistochemistry (IHC).

For immunohistochemistry for L3MBTL2 and RNF8, sections were subjected to antigen retrieval by microwaving in $0.01 \mathrm{M}$ sodium citrate buffer ( $\mathrm{pH}$ 6.0). Sections then were incubated with rabbit anti-L3MBTL2 antibody (HPA000815, Sigma-Aldrich; A08416, Boster Biological Technology) or anti-RNF8 antibody (14112-1-AP, Proteintech) at $4{ }^{\circ} \mathrm{C}$ overnight. The signals were detected using
Histostain Plus LAB-SA Detection System (Invitrogen) or UltraSensitive S-P kit (KIT-9710, MXB Biotechnologies). Sections were counterstained with Harris' hematoxylin. For ubH2A staining, sections were treated with $2 \mathrm{~N} \mathrm{HCl}$ for 30 min at room temperature after antigen retrieval. Sections then were treated with $100 \mathrm{mM}$ Tris- $\mathrm{HCl}(\mathrm{pH} \mathrm{8.0)}$ for 10 min before incubation with anti-ubH2A antibody (\#8240, Cell Signaling).

For immunofluorescence (IF), frozen testis sections were treated with $1 \%$ SDS for 4 min for antigen retrieval. Cauda epididymis sections were treated with $10 \mathrm{mM}$ dithiothreitol (DTT) for $30 \mathrm{~min}$ after microwaving antigen retrieval. Sections then were incubated with primary antibodies and secondary antibodies as indicated (Supplemental Table 1) at $4{ }^{\circ} \mathrm{C}$ overnight, and counterstained with DAPI.

\section{Fertility test}

To test the fertility of L3mbtl 2 cKO male mice, 6-week-old $\mathrm{L} 3 \mathrm{mbtl} 2^{\mathrm{f} / \mathrm{f}}$ or $\mathrm{L} 3 \mathrm{mbtl} 2^{\mathrm{f} / \mathrm{f}}$; Stra8-icre male mice were mated with 6-week-old wild-type C57BL/6 J females. Cages were monitored every 1 or 2 days for the presence and number of pups born. The test was terminated when female mice failed to produce pups for 3 months.

\section{RNA sequencing and data analysis}

Total RNA was extracted from control and L3mbtl2 cKO testes at 8 months of age using RNA extraction kit (Takara) with DNase I treatment. RNA sequencing (RNA-seq) was carried out by the Beijing Genomics Institute (Shenzhen, China) following standard protocols. The library products were sequenced using an Illumina HiSeq 4000 Genome Analyzer. Standard bioinformatics analysis was performed by the Beijing Genomics Institute. The RNA-seq data are available in the National Center for Biotechnology Information Gene Expression Omnibus website (http://www. ncbi.nlm.nih.gov/geo) under accession number GSE109921.

\section{TUNEL assay}

Apoptotic cells in paraffin testis sections were stained with ApopTag Plus Fluorescein In Situ Apoptosis Detection kit (Millipore) following the manufacturer's instructions. The sections were counterstained with DAPI. Apoptotic cell number per tubule was calculated by dividing the total number of TUNEL-positive germ cells by the total number of seminiferous tubules examined for each mouse.

\section{Immunoprecipitation}

HEK293 cells were transfected with HA-RNF8 plasmids in the absence or presence of L3mbtl2-Flag plasmids. Cell 
lysates were incubated with anti-Flag antibody (F1804; Sigma-Aldrich) at $4{ }^{\circ} \mathrm{C}$ overnight. The solutions were then incubated for $6 \mathrm{~h}$ at $4{ }^{\circ} \mathrm{C}$ with protein $\mathrm{G}$ beads (Pierce Biotechnology). Eluted proteins were subjected to Western blotting using anti-HA antibody (ab18181; Abcam). For the reciprocal immunoprecipitation, cells were transfected with L3mbt12-Flag in the absence or presence of HA-RNF8. Cell lysates were precipitated with anti-HA antibody, and analyzed by Western blotting using anti-Flag antibody.

To examine the interaction between L3MBTL2 and RNF8 in the testis, testis lysates were preabsorbed with protein $\mathrm{G}$ beads at $4{ }^{\circ} \mathrm{C}$ overnight. Preabsorbed lysates were incubated with $4 \mu \mathrm{g}$ rabbit RNF8 antibody (ab105362, Abcam) or normal rabbit IgG at $4{ }^{\circ} \mathrm{C}$ overnight. Protein A beads were added, and lysates were incubated at $4{ }^{\circ} \mathrm{C}$ for $6 \mathrm{~h}$. Eluted proteins were subjected to Western blot analysis using anti-L3MBTL2 antibody (HPA000815, Sigma-Aldrich).

\section{Western blotting}

Testes were homogenated and lysed in TBS (Tris-HCl, 50 $\mathrm{mM}, \mathrm{NaCl} 150 \mathrm{mM}, 2 \%$ n-octylglucoside, $\mathrm{pH}$ 7.4) containing protease and phosphatase inhibitors (Pierce Biotechnology) for $30 \mathrm{~min}$ on ice. A total of $25-40 \mu \mathrm{g}$ of protein was separated by SDS-PAGE and transferred to PVDF membranes. Membranes were probed with primary antibodies as indicated (Supplemental Table 1). The signals were developed using ECL western blotting detection reagents (Millipore or GE Healthcare).

To prepare germ cell fractions, whole testis was decapsulated and minced in cold PBS, and the cell mixture then was rotated for $15 \mathrm{~min}$ at $4{ }^{\circ} \mathrm{C}$ and filtered through a $70 \mu \mathrm{M}$ nylon mesh at $1200 \mathrm{~g}$ for $10 \mathrm{~min}$. Whole cell lysate was extracted in NETN buffer $(50 \mathrm{mM}$ Tris- $\mathrm{HCl} \mathrm{pH} 8.0,100 \mathrm{mM}$ $\mathrm{NaCl}, 2 \mathrm{mM}$ EDTA, $0.5 \% \mathrm{NP}-40$ ) for $30 \mathrm{~min}$ on ice. The pellet was separated from the supernatant by centrifuging lysate for $10 \mathrm{~min}$ at $12,000 \mathrm{~g}$, and washed 3 times with PBS. The pellet was then extracted for histones with $0.25 \mathrm{~N} \mathrm{HCl}$.

\section{Meiotic spreads}

Meiotic Spreads were prepared as previously described [43]. Testes were placed in DMEM and the tunicae were removed. The cell suspension was centrifuged for $8 \mathrm{~min}$ at $1000 \mathrm{~g}$, and cell pellet was resuspended in $1 \mathrm{ml}$ of hypotonic buffer (30 mM Tris-HCl pH 8.2, $50 \mathrm{mM}$ sucrose $\mathrm{pH} 8.2,17 \mathrm{mM}$ sodium citrate) and incubated at room temperature for $7 \mathrm{~min}$. Samples were then centrifuged again and resuspended in $0.1 \mathrm{M}$ sucrose. The mixture was spread on slides prepared with $1 \%$ PFA, $0.1 \%$ Triton $\mathrm{X}-100, \mathrm{pH} 9.2$, allowed to dry in a humidified chamber, and stored at $-80{ }^{\circ} \mathrm{C}$. For staining, slides were washed 3 times with PBS, blocked in 3\% BSA for $1 \mathrm{~h}$, and incubated with primary antibodies as indicated (Supplemental Table 1).

\section{Sperm counts}

Caudal epididymides were dissected and immersed in $1 \mathrm{ml}$ M2 medium (Sigma-Aldrich). The tissues were minced to allow the sperm to swim into the medium for $10 \mathrm{~min}$ at $37^{\circ} \mathrm{C}$. The samples were diluted with trypan blue staining solution (Bio-Rad), and were transferred onto a hemocytometer to determine the sperm number.

\section{siRNA knockdown}

Human L3MBTL2 small interfering RNAs (siRNAs) and negative siRNA were purchased from Shanghai GenePharma Co., Ltd (Shanghai, China). A mixture of following two sense L3mbtl2 siRNA sequences were used: $5^{\prime}$ GCUCCAUCGAGACUCUUUATT-3', and 5'-GCCUGCUCUUCCUUAAGAUTT- ${ }^{\prime}$. Hela cells were transfected with control siRNA or L3MBTL2 siRNA mixture. For APH treatment, $38 \mathrm{~h}$ after transfection, cells were treated with and without APH $(0.5 \mu \mathrm{M})$ for $24 \mathrm{~h}$, and cells then were lysed in reducing 2 XSDS sample buffer and subjected to Western blotting. For Zeocin treatment, $48 \mathrm{~h}$ after transfection, cells were treated with or without Zeocin $(100 \mu \mathrm{g} / \mathrm{ml})$ for $1 \mathrm{~h}$ before proteins were extracted for Western blot analysis. For ionizing radiation (IR) treatment, $48 \mathrm{~h}$ after transfection, cells were exposed to $\gamma$-irradiation at $5 \mathrm{~Gy}$ and then incubated for $30 \mathrm{~min}$ at $37^{\circ} \mathrm{C}$.

A mixture of following two sense sequences were used for L3mbtl2 knockdown in mouse GC2 spermatocyte cell line: 5'-GCUCCAGCAAGACUCUUUATT-3', and 5'GCUCCAAGAAACCCUUACUTT- $3^{\prime}$. Scrambled siRNAs from Ambion (AM4613) were used as control.

\section{Nuclear protein extraction}

Cells were collected using hypotonic buffer $(10 \mathrm{mM}$ Hepes, $\mathrm{pH} 7.1 ; 50 \mathrm{mM} \mathrm{NaCl} ; 0.3 \mathrm{M}$ sucrose; $0.1 \%$ Triton $\mathrm{X}-100 ; 0.1 \mathrm{mM}$ EDTA; $1 \mathrm{mM}$ DTT), and incubated for 10 min on ice. Cell nuclei were pelleted by centrifugation at $1500 \mathrm{~g}$, and washed with hypotonic buffer without Triton $\mathrm{X}-100$. Nuclear pellets were resuspended in nuclear extraction buffer (10 mM Hepes, $\mathrm{pH} 7.1 ; 500 \mathrm{mM} \mathrm{NaCl}$; 0.5\% NP-40; $0.1 \mathrm{mM}$ EDTA; $1 \mathrm{mM}$ DTT), and sonicated. Nuclear extracts were obtained after centrifugation at $10,000 \mathrm{~g}$.

\section{Electron microscopy}

Testes and cauda epididymides were trimmed and fixed in $2.5 \%$ glutaraldehyde in $0.1 \mathrm{M}$ sodium phosphate buffer $(\mathrm{pH}$ 
7.2) overnight at $4{ }^{\circ} \mathrm{C}$, postfixed in $2 \%$ osmium tetroxide for $1 \mathrm{~h}$, dehydrated in ascending graded ethanol, and embedded in EPON 812 at $60{ }^{\circ} \mathrm{C}$ for $24 \mathrm{~h}$. Ultrathin $60 \mathrm{~nm}$ thick sections were stained with uranyl acetate and lead citrate and examined in a Hitachi H-7700 transmission electron microscope.

\section{Statistical analysis}

The data presented are means \pm SD of independent replicates $(n \geq 3)$. Student's $t$ test was applied for statistical analysis. Mann-Whitney $U$ test was used to analyze the differences in MLH foci between WT and cKO. $p$ values $\leq 0.05$ were considered statistically significant.

Acknowledgements We are grateful to Dr. P. Jeremy Wang (University of Pennsylvania) for helpful suggestion and critical reading and editing of the manuscript. We thank Josie Lai and Jean Kung (The Chinese University of Hong Kong) for technical assistance with the electronic microscopy. We also thank Dr. Wenhui Zhao (Peking University) for the CMV-HA-RNF8 plasmid, and Dr. Winnie Shum (ShanghaiTech University) for helpful discussions. Mr. Geng Tian (Shanghai Jiao Tong University) helped with the bioinformatics analysis. This work was supported by Health and Medical Research Fund (HMRF) grants 04153586 and 05161376 (to Y. X.).

\section{Compliance with ethical standards}

Conflict of interest The authors declare that they have no conflict of interest.

Publisher's note: Springer Nature remains neutral with regard to jurisdictional claims in published maps and institutional affiliations.

\section{References}

1. Hess RA, Renato de Franca L. Spermatogenesis and cycle of the seminiferous epithelium. Adv Exp Med Biol. 2008;636:1-15.

2. Mahadevaiah SK, Turner JM, Baudat F, Rogakou EP, de Boer P, Blanco-Rodriguez J, et al. Recombinational DNA double-strand breaks in mice precede synapsis. Nat Genet. 2001;27:271-6.

3. Turner JM, Aprelikova O, Xu X, Wang R, Kim S, Chandramouli $\mathrm{GV}$, et al. BRCA1, histone $\mathrm{H} 2 \mathrm{AX}$ phosphorylation, and male meiotic sex chromosome inactivation. Curr Biol. 2004;14:2135-42.

4. Turner JM, Mahadevaiah SK, Fernandez-Capetillo O, Nussenzweig A, Xu X, Deng CX, et al. Silencing of unsynapsed meiotic chromosomes in the mouse. Nat Genet. 2005;37:41-7.

5. Garcia-Cruz R, Roig I, Caldes MG. Maternal origin of the human aneuploidies. Are homolog synapsis and recombination to blame? Notes (learned) from the underbelly. Genome Dyn. 2009;5:128-36.

6. Blanco-Rodriguez J. Programmed phosphorylation of histone $\mathrm{H} 2 \mathrm{AX}$ precedes a phase of DNA double-strand break-independent synapsis in mouse meiosis. Reproduction. 2012;144:699-712.

7. Balhorn R. A model for the structure of chromatin in mammalian sperm. J Cell Biol. 1982;93:298-305.

8. Baarends WM, Hoogerbrugge JW, Roest HP, Ooms M, Vreeburg J, Hoeijmakers JH, et al. Histone ubiquitination and chromatin remodeling in mouse spermatogenesis. Dev Biol. 1999;207:322-33.

9. Hazzouri M, Pivot-Pajot C, Faure AK, Usson Y, Pelletier R, Sele $\mathrm{B}$, et al. Regulated hyperacetylation of core histones during mouse spermatogenesis: involvement of histone deacetylases. Eur J Cell Biol. 2000;79:950-60.

10. Godmann M, Auger V, Ferraroni-Aguiar V, Di Sauro A, Sette C, Behr R, et al. Dynamic regulation of histone H3 methylation at lysine 4 in mammalian spermatogenesis. Biol Reprod. 2007;77:754-64.

11. Moriniere J, Rousseaux S, Steuerwald U, Soler-Lopez M, Curtet S, Vitte AL, et al. Cooperative binding of two acetylation marks on a histone tail by a single bromodomain. Nature. 2009;461:664-8.

12. Montellier E, Boussouar F, Rousseaux S, Zhang K, Buchou T, Fenaille $\mathrm{F}$, et al. Chromatin-to-nucleoprotamine transition is controlled by the histone H2B variant TH2B. Genes Dev. 2013;27:1680-92.

13. Lu LY, Wu J, Ye L, Gavrilina GB, Saunders TL, Yu X. RNF8dependent histone modifications regulate nucleosome removal during spermatogenesis. Dev Cell. 2010;18:371-84.

14. Gou LT, Kang JY, Dai P, Wang X, Li F, Zhao S, et al. Ubiquitination-deficient mutations in human piwi cause male infertility by impairing histone-to-protamine exchange during spermiogenesis. Cell. 2017;169:1090-104 e1013.

15. Bonasio R, Lecona E, Reinberg D. MBT domain proteins in development and disease. Semin Cell Dev Biol. 2010;21:221-30.

16. Qin J, Whyte WA, Anderssen E, Apostolou E, Chen HH, Akbarian $\mathrm{S}$, et al. The polycomb group protein L3mbtl2 assembles an atypical PRC1-family complex that is essential in pluripotent stem cells and early development. Cell Stem Cell. 2012;11:319-32.

17. Wismar J, Loffler T, Habtemichael N, Vef O, Geissen M, Zirwes $\mathrm{R}$, et al. The Drosophila melanogaster tumor suppressor gene lethal(3)malignant brain tumor encodes a proline-rich protein with a novel zinc finger. Mech Dev. 1995;53:141-54.

18. Wismar J. Molecular characterization of h-l(3)mbt-like: a new member of the human mbt family. FEBS Lett. 2001;507:119-21.

19. Ogawa H, Ishiguro K, Gaubatz S, Livingston DM, Nakatani Y. A complex with chromatin modifiers that occupies E2F- and Mycresponsive genes in G0 cells. Science. 2002;296:1132-6.

20. Trojer P, Li G, Sims RJ 3rd, Vaquero A, Kalakonda N, Boccuni P, et al. L3MBTL1, a histone-methylation-dependent chromatin lock. Cell. 2007;129:915-28.

21. Frankenberg S, Smith L, Greenfield A, Zernicka-Goetz M. Novel gene expression patterns along the proximo-distal axis of the mouse embryo before gastrulation. BMC Dev Biol. 2007;7:8.

22. Trojer P, Cao AR, Gao Z, Li Y, Zhang J, Xu X, et al. L3MBTL2 protein acts in concert with PcG protein-mediated monoubiquitination of $\mathrm{H} 2 \mathrm{~A}$ to establish a repressive chromatin structure. Mol Cell. 2011;42:438-50.

23. Bergink S, Salomons FA, Hoogstraten D, Groothuis TA, de Waard $\mathrm{H}, \mathrm{Wu} \mathrm{J}$, et al. DNA damage triggers nucleotide excision repair-dependent monoubiquitylation of histone H2A. Genes Dev. 2006;20:1343-52.

24. Chou DM, Adamson B, Dephoure NE, Tan X, Nottke AC, Hurov $\mathrm{KE}$, et al. A chromatin localization screen reveals poly (ADP ribose)-regulated recruitment of the repressive polycomb and NuRD complexes to sites of DNA damage. Proc Natl Acad Sci USA. 2010;107:18475-80.

25. Facchino S, Abdouh M, Chatoo W, Bernier G. BMI1 confers radioresistance to normal and cancerous neural stem cells through recruitment of the DNA damage response machinery. J Neurosci. 2010;30:10096-111.

26. Ismail IH, Andrin C, McDonald D, Hendzel MJ. BMI1-mediated histone ubiquitylation promotes DNA double-strand break repair. J Cell Biol. 2010;191:45-60.

27. Ginjala V, Nacerddine K, Kulkarni A, Oza J, Hill SJ, Yao M, et al. BMI1 is recruited to DNA breaks and contributes to DNA damage-induced H2A ubiquitination and repair. Mol Cell Biol. 2011;31:1972-82. 
28. Leung JW, Agarwal P, Canny MD, Gong F, Robison AD, Finkelstein IJ, et al. Nucleosome acidic patch promotes RNF168- and RING1B/BMI-dependent $\mathrm{H} 2 \mathrm{AX}$ and $\mathrm{H} 2 \mathrm{~A}$ ubiquitination and DNA damage signaling. PLoS Genet. 2014;10:e1004178.

29. Huang $\mathrm{H}, \mathrm{Xu} \mathrm{C}$, Wang Y, Meng C, Liu W, Zhao Y, et al. Lethal (3) malignant brain tumor-like 2 (L3MBTL2) protein protects against kidney injury by inhibiting the DNA damage-p53apoptosis pathway in renal tubular cells. Kidney Int. 2018;93: $855-70$

30. Takada Y, Isono K, Shinga J, Turner JM, Kitamura H, Ohara O, et al. Mammalian Polycomb Scmh1 mediates exclusion of Polycomb complexes from the XY body in the pachytene spermatocytes. Development. 2007;134:579-90.

31. Hasegawa K, Sin HS, Maezawa S, Broering TJ, Kartashov AV, Alavattam KG, et al. SCML2 establishes the male germline epigenome through regulation of histone $\mathrm{H} 2 \mathrm{~A}$ ubiquitination. Dev Cell. 2015;32:574-88.

32. Luo M, Zhou J, Leu NA, Abreu CM, Wang J, Anguera MC, et al. Polycomb protein SCML2 associates with USP7 and counteracts histone $\mathrm{H} 2 \mathrm{~A}$ ubiquitination in the XY chromatin during male meiosis. PLoS Genet. 2015;11:e1004954.

33. Handel MA, Schimenti JC. Genetics of mammalian meiosis: regulation, dynamics and impact on fertility. Nat Rev Genet. 2010;11:124-36

34. Lu LY, Xiong Y, Kuang H, Korakavi G, Yu X. Regulation of the DNA damage response on male meiotic sex chromosomes. Nat Commun. 2013;4:2105.

35. Pittman DL, Cobb J, Schimenti KJ, Wilson LA, Cooper DM, Brignull E, et al. Meiotic prophase arrest with failure of chromosome synapsis in mice deficient for Dmc1, a germline-specific RecA homolog. Mol Cell. 1998;1:697-705.
36. Moens PB, Kolas NK, Tarsounas M, Marcon E, Cohen PE, Spyropoulos B. The time course and chromosomal localization of recombination-related proteins at meiosis in the mouse are compatible with models that can resolve the early DNA-DNA interactions without reciprocal recombination. J Cell Sci. 2002;115(Pt 8):1611-22.

37. Adams SR, Maezawa S, Alavattam KG, Abe H, Sakashita A, Shroder M, et al. RNF8 and SCML2 cooperate to regulate ubiquitination and $\mathrm{H} 3 \mathrm{~K} 27$ acetylation for escape gene activation on the sex chromosomes. PLoS Genet. 2018;14:e1007233.

38. Mailand N, Bekker-Jensen S, Faustrup H, Melander F, Bartek J, Lukas C, et al. RNF8 ubiquitylates histones at DNA double-strand breaks and promotes assembly of repair proteins. Cell. 2007;131:887-900.

39. Nowsheen S, Aziz K, Aziz A, Deng M, Qin B, Luo K, et al. L3MBTL2 orchestrates ubiquitin signalling by dictating the sequential recruitment of RNF8 and RNF168 after DNA damage. Nat Cell Biol. 2018;20:455-64.

40. Burgoyne PS, Mahadevaiah SK, Turner JM. The consequences of asynapsis for mammalian meiosis. Nat Rev Genet. 2009;10:207-16.

41. Wang H, Wang L, Erdjument-Bromage H, Vidal M, Tempst P, Jones RS, et al. Role of histone H2A ubiquitination in Polycomb silencing. Nature. 2004;431:873-8.

42. Sadate-Ngatchou PI, Payne CJ, Dearth AT, Braun RE. Cre recombinase activity specific to postnatal, premeiotic male germ cells in transgenic mice. Genesis. 2008;46:738-42.

43. Peters AH, Plug AW, van Vugt MJ, de Boer P. A drying-down technique for the spreading of mammalian meiocytes from the male and female germline. Chromosome Res. 1997;5:66-8.

\section{Affiliations}

\section{Chenling Meng ${ }^{1} \cdot$ Jinyue Liao ${ }^{1} \cdot$ Danfeng Zhao ${ }^{2} \cdot$ Huihui Huang ${ }^{1} \cdot$ Jinzhong Qin ${ }^{3} \cdot$ Tin-Lap Lee $^{1} \cdot$ Degui Chen $^{2} \cdot$ Wai-Yee Chan ${ }^{1,4,5} \cdot$ Yin Xia $\left(^{1,4,5}\right.$}

1 School of Biomedical Sciences, Faculty of Medicine, The Chinese University of Hong Kong, Hong Kong, China

2 State Key Laboratory of Molecular Biology, Shanghai Key Laboratory of Molecular Andrology, Institute of Biochemistry and Cell Biology, Shanghai Institutes for Biological Sciences, Chinese Academy of Sciences, 200031 Shanghai, China

3 The Key Laboratory of Model Animals for Disease Study of Ministry of Education, Model Animal Research Center, Nanjing
University, Nanjing, China

4 Key Laboratory for Regenerative Medicine (Jinan University-The Chinese University of Hong Kong), Ministry of Education, School of Biomedical Sciences, Faculty of Medicine, The Chinese University of Hong Kong, Hong Kong, China

5 School of Biomedical Sciences Core Laboratory, The Chinese University of Hong Kong Shenzhen Research Institute, Shenzhen, China 